\title{
Prenatal diagnosis of sex chromosome mosaicism with two marker chromosomes in three cell lines and a review of the literature
}

\author{
JIANLI ZHENG ${ }^{1}$, XIAOYU YANG ${ }^{2}$, HAIYAN LU ${ }^{1}$, YONGJUAN GUAN ${ }^{1}$, FANGFANG YANG ${ }^{1}$,

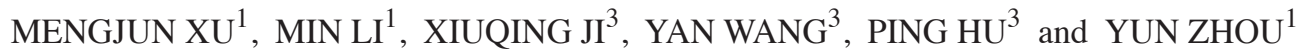 \\ ${ }^{1}$ Department of Prenatal Diagnosis, Laboratory of Clinical Genetics, Maternity and Child Health Care Hospital, \\ Yancheng, Jiangsu 224001; ${ }^{2}$ Department of Clinical Reproductive Medicine, State Key Laboratory of \\ Reproductive Medicine, The First Affiliated Hospital of Nanjing Medical University, Nanjing, Jiangsu 210029; \\ ${ }^{3}$ Department of Prenatal Diagnosis, State Key Laboratory of Reproductive Medicine, \\ Obstetrics and Gynecology Hospital Affiliated to Nanjing Medical University, Nanjing, Jiangsu 210004, P.R. China
}

Received March 31, 2018; Accepted November 21, 2018

DOI: $10.3892 / \mathrm{mmr} .2018 .9798$

\begin{abstract}
The present study described the diagnosis of a fetus with sex chromosome mosaicism in three cell lines and two marker chromosomes. A 24-year-old woman underwent amniocentesis at 21 weeks and 4 days of gestation due to noninvasive prenatal testing identifying that the fetus had sex chromosome abnormalities. Amniotic cell culture revealed a karyotype of 45,X[13]/46,X,+mar1[6]/46,X,+mar2[9], and prenatal ultrasound was unremarkable. The woman underwent repeat amniocentesis at 23 weeks and 4 days of gestation for molecular detection. Single nucleotide polymorphism (SNP) microarray analysis on uncultured amniocytes revealed that the fetus had two $\mathrm{Y}$ chromosomes and 7.8-Mb deletions in Yq11.222q12. The deletion regions included DAZ, RBMY and PRY genes, which could cause spermatogenesis obstacle and sterility. Interphase fluorescence in situ hybridization (FISH) using centromeric probes DXZ1/DYZ3/D18Z1 was performed on uncultured amniocytes to verify the two marker chromosomes to be $\mathrm{Y}$ chromosome derivatives. According to these examinations, the marl was identified as a derivative of the $\mathrm{Y}$ chromosome with a deletion in Yq11.222q12, and the mar2 was identified as a dicentric derivative of the $\mathrm{Y}$ chromosome. The molecular karyotype was therefore 45,X, ish(DXZ1+, DYZ3-,D18Z1++)[5]/46,X,del(Y)(q11.222),ish(DXZ1+,DYZ3+, D18Z1++)[11]/46, X,idic(Y)(q11.222),ish(DXZ1+,DYZ3++, D18Z1++)[14]. The comprehensive use of cytogenetic, SNP array and FISH detections was advantageous for accurately
\end{abstract}

Correspondence to: Dr Jianli Zheng or Dr Haiyan Lu, Department of Prenatal Diagnosis, Laboratory of Clinical Genetics, Maternity and Child Health Care Hospital, 34 Yulong West Road, Yancheng, Jiangsu 224001, P.R. China

E-mail: zj1-0221@163.com

E-mail: 1hyycfy@163.com

Key words: sex chromosome, mosaicism, marker chromosome, isodicentric $\mathrm{Y}$ chromosome, prenatal diagnosis identifying the karyotype, identifying the origin of the marker chromosome and preparing effective genetic counseling.

\section{Introduction}

Abnormalities involving sex chromosomes account for approximately $0.5 \%$ of live births. Individuals with mosaic structural aberrations of the $\mathrm{X}$ and $\mathrm{Y}$ chromosomes exhibit complicated and variable phenotypes. The phenotypes of sex chromosome mosaicism vary from females with Turner syndrome to males with infertility, and include individuals with ambiguous genitalia (1).

Cytogenetically visible Y chromosome anomalies involve deletions, translocations, rings and isochromosomes among others. The $\mathrm{Y}$ chromosome comprises highly repetitive sequences, including direct repeats, inverted repeats and palindromes (2). The highly repetitive structures of the $\mathrm{Y}$ chromosome are mainly located in the azoospermia factor (AZF) regions, where rearrangements occur in high frequency to form delY, idicY and so forth. These rearrangements lead not only to loss, but also gain of specific genes $(3,4)$. The locus for AZF in Yq11.2 is subdivided into the AZFa, AZFb and AZFc regions, which serve an important role in spermatogenesis and fertility (5).

Notably, the majority of Y chromosome anomalies have been reported in a mosaic form, usually in association with a $45, X$ cell line $(6,7)$. In the process of gonadal development, the percentage of 45 cells has an important role in sex determination. A dicentric $\mathrm{Y}$ chromosome is a common abnormal structural rearrangement between sister chromatids and is unstable during cell division; therefore, it is highly likely to generate various cell lines, including $45, \mathrm{X}$ and delY cell lines. Previous studies have mostly described individuals with ambiguous genitalia and mixed gonadal dysgenesis who were diagnosed with sex chromosome mosaicism postnatally (1,8-16). However, there are few studies reporting prenatally diagnosed fetuses, particularly with three different cell lines.

The present study reports the case of a fetus with a 45,X/46,X,del(Y)(q11.222)/46,X,idic(Y)(q11.222) karyotype. The fetus carried a derivative $\mathrm{Y}$ chromosome with 
the deletion of $\mathrm{AZFb}$ and $\mathrm{AZFc}$ regions, and a dicentric $\mathrm{Y}$ chromosome with a break point located in the Yq11.222. The results were confirmed through cytogenetic, single nucleotide polymorphism (SNP) microarray and fluorescence in situ hybridization (FISH) detections. The comprehensive use of multiple technologies was beneficial for accurately diagnosing the karyotype, identifying the origin of the marker chromosome and preparing effective genetic counseling.

\section{Materials and methods}

Case report. A 24-year-old pregnant female (gravida 1 para 0 ) was enrolled into the present study. The patient experienced regular menstruation (4 days per 28 days), was in good health, had no abnormal family history, and had not been exposed to teratogenic agents prior to or during the pregnancy. She refused serum screening and underwent noninvasive prenatal testing (NIPT) at 16 weeks and 4 days of gestation. The NIPT results suggested suspicious abnormalities of the fetal sex chromosomes. Subsequently, amniocentesis was performed at 21 weeks and 4 days of gestation, and 20-ml amniotic fluid was sampled under ultrasound guidance for routine amniotic fluid cell culture and karyotype analysis. The karyotype analysis results indicated that the fetus exhibited sex chromosome mosaicism with two marker chromosomes. Therefore, further assays were recommended. Amniotic fluid $(20 \mathrm{ml})$ was sampled again at 23 weeks and 4 days of gestation for analysis by SNP microarray and FISH assay. The molecular results confirmed the existence of two Y chromosomes, deletions in $\mathrm{Yq}$ and mosaicism. The patient terminated the pregnancy at 27 weeks of gestation. Karyotype analysis of peripheral blood samples obtained from the father and mother of the fetus was identified as 46,XY and 46,XX, respectively. This study was approved by the Medical Ethics Committee of the Maternity and Child Health Care Hospital (Yancheng, China), and written informed consent was obtained from the two participants.

Cytogenetics. Amniotic fluid cell culture was performed according to standard techniques (17). Routine G-bands were obtained using trypsin and Giemsa staining (GTG analysis) at 400 band resolution, and this analysis was used to prepare the amniotic cell chromosome specimens. The peripheral blood lymphocytes obtained from the parents were cultured, followed by karyotyping analysis. GTG-banding was also performed according to the standard protocol.

FISH analysis. In order to ascertain the origins of the marker chromosomes and mosaic proportions, FISH analysis was performed on the amniotic specimens using the DXZ1/DYZ3/D18Z1 probes located at the centromeric region of chromosomes X, Y and 18 (Vysis; Abbott Laboratories, Abbott Park, IL, USA), according to the manufacturer's protocol. Human chromosomes were stained by 4',6-diamidino-2-phenylindole (Vysis; Abbott Laboratories) in the dark for 10-15 min at room temperature and exhibited bright fluorescence at the desired locations for detection.

SNP microarray. Human cyto12 SNP-array (Illumina, Inc., San Diego, CA, USA) comprising around 300,000 SNP probes was applied to perform a whole genome scan on the amniotic cell DNA of the fetus. SNP-array tests were performed according to the manufacturer's protocol (Illumina, Inc.), while molecular karyotype analysis was conducted using KaryoStudio version 1.4.3.0 (Illumina, Inc.). Databases including DECIPHER (http://decipher.sanger.ac.uk/), DGV (http://projects.tcag.ca/variation), UCSC (http://genome.ucsc. edu/) and OMIM (http://www.ncbi.nlm.nih.gov/omim), were used as references to evaluate the array data and analyze genotype-phenotype correlations.

\section{Results}

Karyotype analysis. The routine G-band staining analysis indicated that the fetus had a mosaic karyotype with two marker chromosomes in three cell lines. The abnormal karyotype was 45,X[13]/46,X,+mar1[6]/46,X,+mar2[9], and the mosaic proportion was 46, 21 and $33 \%(13 / 28,6 / 28$ and 9/28 cells), respectively (Fig. 1A-C). GTG analysis was also performed for the parents, who were both revealed to have a normal karyotype.

FISH analysis. The DXZ1/DYZ3/D18Z1 probes were successfully hybridized to interphase cell nuclei. There was one signal and two signals in the centromeric regions of chromosomes X and 18, respectively (Fig. 1D-F). As displayed in Fig. 1D, there was no Y centromeric signal. One red signal revealed one Y centromere of marl (Fig. 1E), and two signals revealed two Y centromeres of mar2 (Fig. 1F). In addition, FISH analysis on uncultured amniocytes revealed the karyotype 45,X,ish(DXZ1+,DYZ3-,D18Z1++)[5]/46,X,+mar1,ish (DXZ1+,DYZ3+,D18Z1++)[11]/46,X,+mar2,ish(DXZ1+,DYZ $3++, \mathrm{D} 18 \mathrm{Z} 1++)[14]$. The mosaic proportion of this karyotype sequence was 17,37 and $46 \%$ (5/30, 11/30 and 14/30 cells), respectively.

SNP microarray. The SNP microarray analysis performed on the DNA extracted from amniotic fluid revealed two $\mathrm{Y}$ chromosomes and 7.8-Mb deletions involving the region in Yq11.222q12. The molecular karyotype was $\operatorname{arr}$ [hg19] (X)x1, (Y)x2, Yq11.222q12(21,032,051-28,786,812)x0 (Fig. 2A and B). The deletion parts were located at AZFb and $\mathrm{AZFc}$ regions in the long arm of the $\mathrm{Y}$ chromosome, including a number of OMIM genes, such as the CDY, HSFY, RBMY, PRY, BPY2 and DAZ gene families (Fig. 3).

Prenatal diagnosis. Combining the results of chromosome karyotype analysis, FISH and SNP-array analysis, it was possible to identify the precise breakage of the marl and mar2 on Yq11.222. According to these results, the molecular karyotype of the fetus was identified as 45,X,ish(DXZ1+,DYZ3-,D18Z1++)[5]/46,X, $\operatorname{del}(Y)(q 11.222), i s h(D X Z 1+, D Y Z 3+, D 18 Z 1++)[11] / 46, X, \operatorname{idic}(Y)$ (q11.222), ish(DXZ1+,DYZ3++,D18Z1++)[14]. SNP-array analysis revealed two $\mathrm{Y}$ chromosomes and 7.8-Mb deletions in Yq11.222q12 located at AZFb and AZFc regions. The deletion regions included DAZ, RBMY and PRY genes, which could cause spermatogenesis obstacle and sterility. Although the proportions of $45, \mathrm{X}$ cell lines were discrepant between the karyotype and FISH analysis results, it could serve a role in sex determination and gonad development. Following genetic 

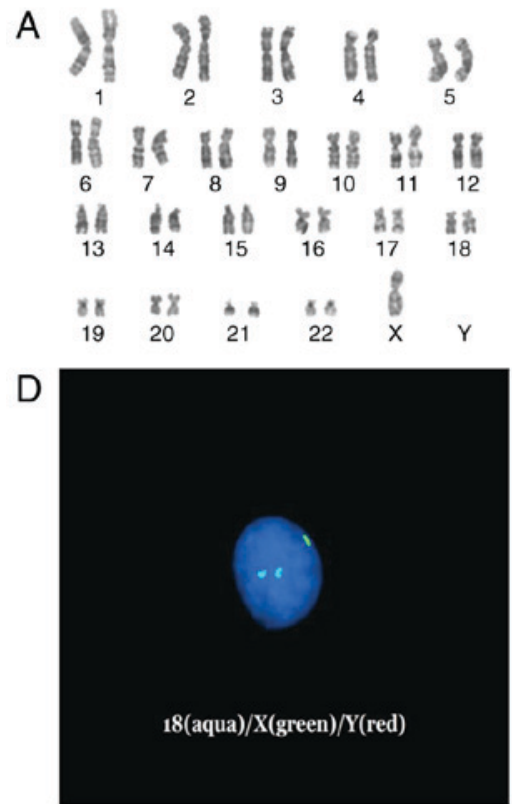

B
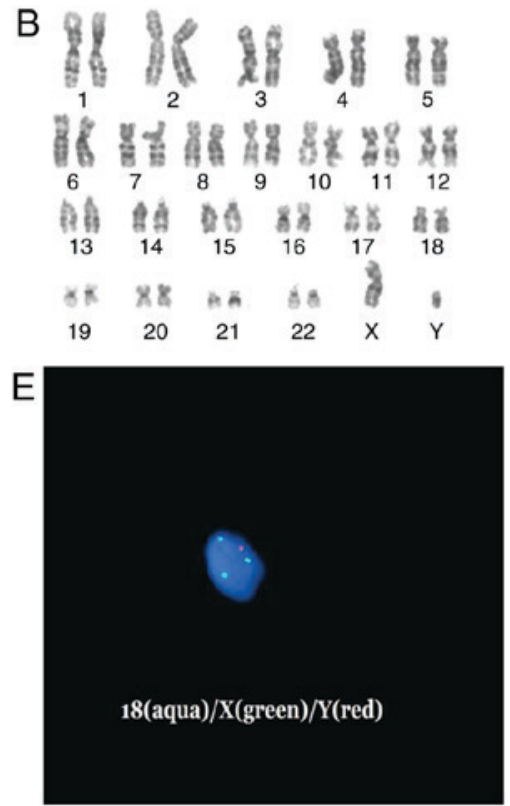

C
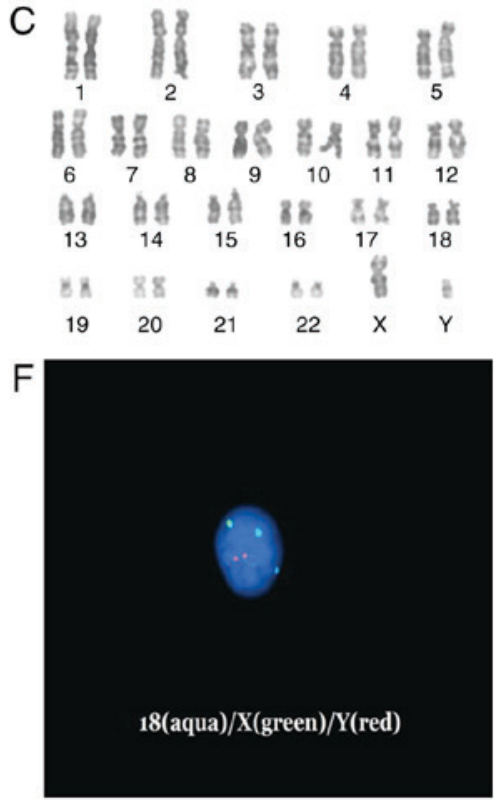

Figure 1. (A-C) Giemsa banding karyotype analysis of the fetus: (A) 45,X (B) 46,X,del(Y)(q11.222) (C) 46,X,idic(Y)(q11.222) and (D-F) D18Z1(aqua)/DXZ1(green)/DYZ3(red) centromeric probe detection in interphase cell nuclei of the fetus: (D) Two aqua fluorescent signals indicate two chr18, one green signal indicates one chrX. (E) Two aqua signals indicate two chr18, one green signal indicates one chrX, one red signal indicates one chrY. (F) Two aqua signals indicate two chr18, one green signal indicates one chrX, two red signals indicate idicY (combined with the karyotype C). Chr, chromosome.

consultation, the parents decided to terminate the pregnancy, and labor was induced at 27 weeks of gestation.

\section{Discussion}

In the present study, a fetus was found to have a mosaic karyotype of three cell lines 45,X[13]/46,X,+mar1[6]/46,X,+mar2[9] using the GTG method, while FISH and SNP-array analyses were then conducted to identify the mosaic percentage and origins of the two marker chromosomes. The mosaic percentage differences between the karyotype and FISH analysis results may be caused by the amniotic cell culture selective growth and cell counting $(18,19)$. Although SNP-array analysis had a high level of resolution, low mosaicism could not be detected with this analysis. In addition, it was ascertained that marl was $\operatorname{del}(\mathrm{Y})(\mathrm{q} 11.222)$ with a 7.8-Mb deletion from Yq11.222 to the long-arm end and mar2 was $\operatorname{idic}(Y)(q 11.222)$ with two mar1s connected in Yq11.222 with two Y centromeres.

The abnormal Y chromosomes of the fetus were a result of rearrangements at the meiosis phase between sister chromatids or intrachromosome during spermatogenesis in the father. Another cause probably occurred during the mitosis stages (20). Structural aberrations of the Y chromosome result in predisposition to subsequent chromosome instability and loss of the abnormal $\mathrm{Y}$ chromosome, leading to mosaic 45,X. A fetus with the presence of a $45, X$ cell line has a risk of being a phenotypic female with Turner syndrome manifestations or having ambiguous external genitalia, whether the other cell line is Yp, Yq, Yp plus Yq, or even a free Y chromosome $(6,20)$.

Although to the best of our knowledge there are no identical case reports in the literature, there are a number of cases with 45,X/46,X,idic(Y) and 45,X/46,X,del(Y)(q11.2). Phenotypes associated with the two sex chromosomal mosaicism vary from females with Turner syndrome to males with infertility, and include individuals with ambiguous genitalia (1). Their phenotypic spectrums are very broad and variable, and are attributed to variable locations of the breakpoints and to the proportion of $45, \mathrm{X}$ cells distributed over the different tissues $(9,21,22)$.

As in the majority of previous studies with idicY and delY $(1,5,7,8,12-16)$, the breakpoint in the fetus is in the long arm of chromosome $\mathrm{Y}$, which results in the duplication of the entire short arm and centromere, and a deletion of the distal Yq. A review by Hsu (6) reported 74 cases with mos45,X/46,X,idic(Y)(q11). Among them, 20 cases (27\%) involved phenotypic males with abnormal testes and azoospermia, 17 cases (23\%) had ambiguous external genitalia with mixed gonads and short height, and 37 cases (50\%) were phenotypic females with streak gonads and Ullrich-Turner syndrome changes. The aforementioned review also reported 38 postnatal cases of mos45,X/46,X,del(Y)(q11) manifesting as phenotypic males with hypospadias and azoospermia in 13 cases $(34.2 \%)$, intersex individuals with ambiguous external genitalia in 18 cases $(47.4 \%)$ and phenotypic females with streak gonads and short stature in 7 cases (18.4\%) (6).

To date, few prenatal cases of the karyotype $45, X / 46, X, \operatorname{idic}(Y)(q 11)$ and 45,X/46,X,del(Y)(q11.2) have been reported. Telvi et al (23) reported substantial differences between prenatally and postnatally diagnosed cases of 45,X/46,XY mosaicism. A normal male phenotype was detected in $90 \%$ of prenatally diagnosed cases, whereas the postnatally diagnosed cases exhibited a wide spectrum of phenotypes. This $10 \%$ risk of an abnormal outcome in prenatally diagnosed cases requires further attention (23). In the present study, the prenatal ultrasound of the fetus was unremarkable, because the symptoms of sex chromosome mosaicism would be clinically evident following birth or puberty. 


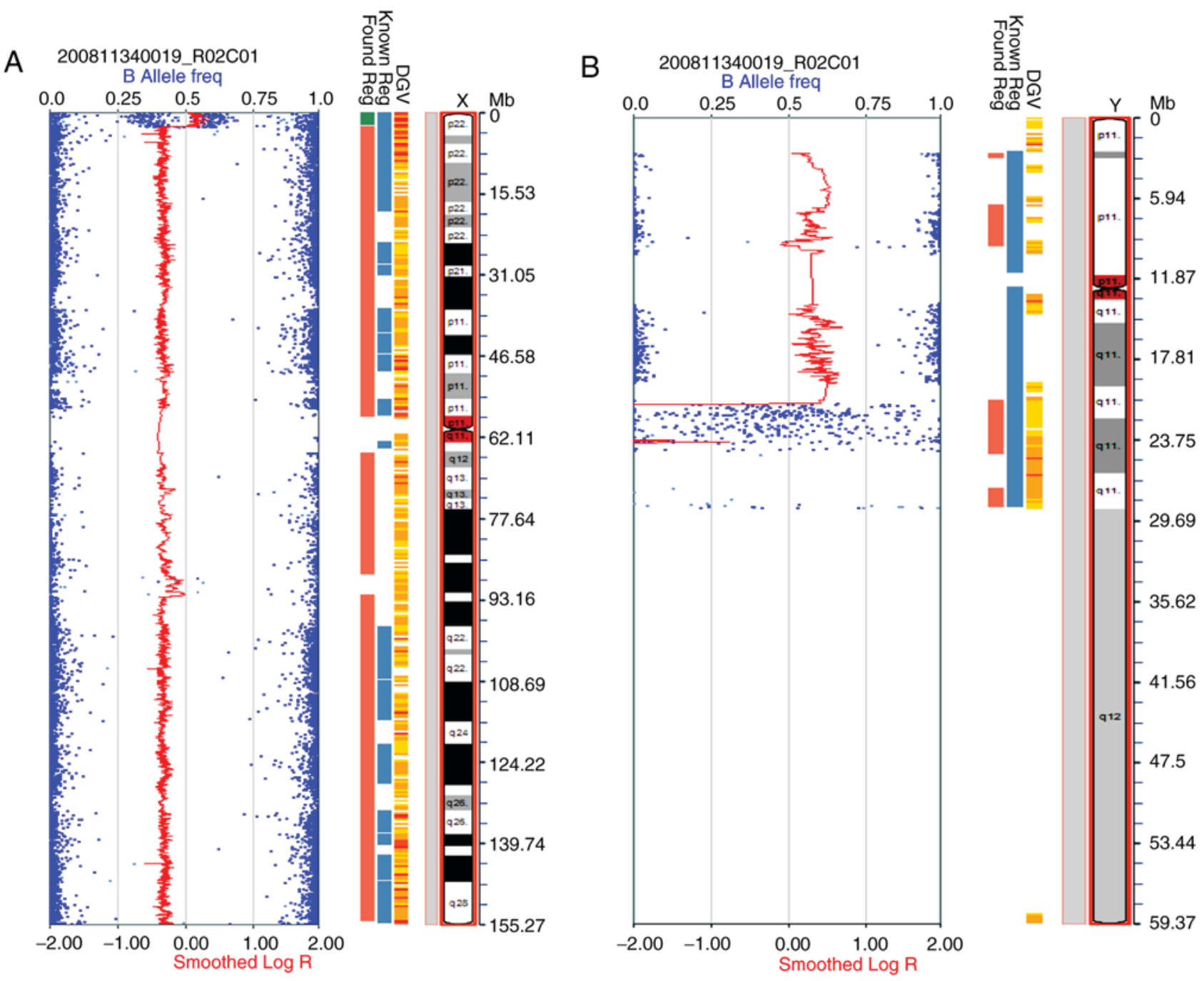

Figure 2. Results of single nucleotide polymorphism array assay, identifying that the molecular karyotype was arr[hg19] (X)X1, (Y)x2, Yq11. 222q12(21,032,051-28,786,812)x0. (A) X chromosome and (B) two Y chromosomes containing 7.8-Mb deletions between Yq11.222 and q12.

A
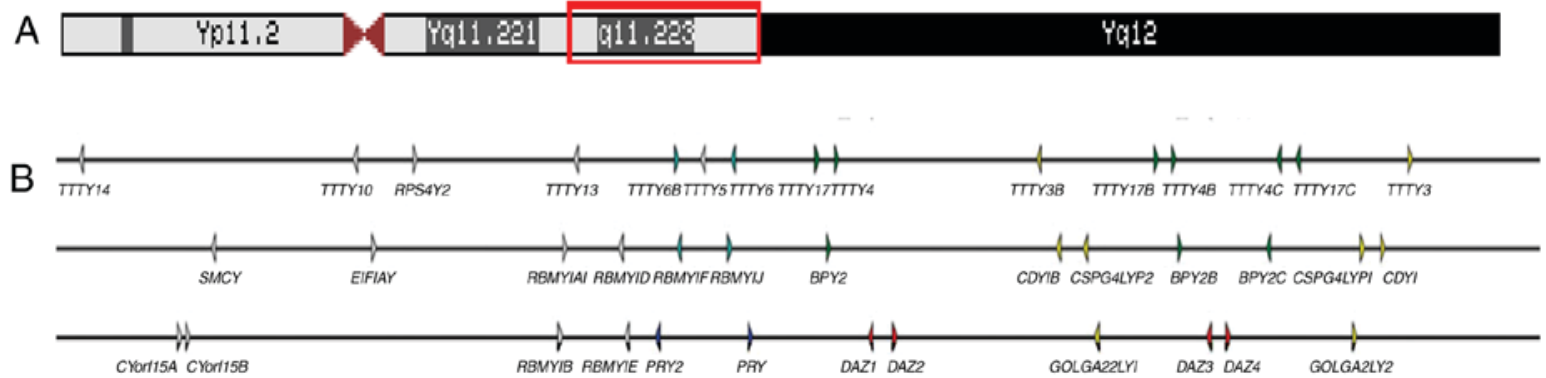

Figure 3. (A) Schematic representation of Y chromosome. The red box indicates the deletion region. (B) Analysis of the genomic location of breakpoints, as determined by the UCSC genome browser.

The $\mathrm{Y}$ chromosome is the shortest chromosome of the human genome, but accumulates male-related genes, including the sex-determining region of Y-chromosome (SRY) and several spermatogenesis-associated genes. The long arm of chromosome $\mathrm{Y}$ is enriched with palindromes that have recently been demonstrated to mediate rearrangements between the arms of sister chromatids (24). The rearrangements lead to not only loss, but also gain of specific genes. Loss of Y-chromosome sequences has been detected in men with azoospermia or severe oligospermia, leading to the definition of the azoospermia factor region. The region has been mapped to Yq11.22-23 and consists of three sub-regions termed AZFa, $\mathrm{AZFb}$ and $\mathrm{AZFc}$ (3-5). Gain of Y-chromosome sequences may generate the idicYp chromosomes and other attachments.

In the current study, the idicY chromosome of the fetus had two SRY genes. The deletion parts of the fetus were located at $\mathrm{AZFb}$ and $\mathrm{AZFc}$ regions in the long arm of $\mathrm{Y}$ chromosome, including the OMIM genes of the CDY, HSFY, RBMY1, PRY, 
BPY2 and DAZ gene families. A study by Lahn and Page (25) identified the CDY, PRY and BPY2 testes-specific gene families. Furthermore, Skaletsky et al (26) determined that the HSFY gene is exclusively expressed in the testes, and there are two palindromic copies of the HSFY gene within palindrome 4 of chromosome Yq. A literature review on the RBMY1 gene family reported by Delbridge et al (27) indicated that the RBMY1 gene family may have an additional role in germ cell development. The DAZ gene encodes an RNA-binding protein with a role in spermatogenesis (28). Taken together, the deletion regions can cause spermatogenesis obstacle and sterility.

In conclusion, multiple studies have observed that patients with $45, \mathrm{X} / 46, \mathrm{X}, \operatorname{idic}(\mathrm{Y})(\mathrm{q} 11)$ or $45, \mathrm{X} / 46, \mathrm{X}, \operatorname{del}(\mathrm{Y})$ (q11.2) exhibit ambiguous external genitalia, azoospermia or Turner syndrome (8-16). Therefore, it is likely that the fetus in the present study would suffer similar syndromes upon maturation. The comprehensive use of cytogenetic, SNP-array and FISH detections in the current study provided adequate genetic counseling to the patient and her family, and the family decided to terminate the pregnancy as the fetus would have been born with birth defects. Thus, the present study may provide guidance for future pregnancy and birth decisions.

\section{Acknowledgements}

Not applicable.

\section{Funding}

No funding was received for this study.

\section{Availability of data and materials}

All data generated or analyzed during this study are included in the article.

\section{Authors' contributions}

JZ drafted the paper and interpreted the SNP-array and FISH data. XY drafted part of the discussion and interpreted the cytogenetic and molecular data. YG, FY and MX were responsible for the conventional cytogenetic analysis. ML and YZ collected the data and provided clinical consultation. XJ, YW and $\mathrm{PH}$ were involved in the SNP-array and FISH analysis. JZ and HL designed the study and gave the final approval of the manuscript. All of the authors read and approved the final manuscript.

\section{Ethics approval and consent to participate}

This study was approved by the Medical Ethics Committee of Maternity and Child Health Care Hospital (Yancheng, China), and written informed consent was obtained from the two participants.

\section{Patient consent for publication}

Not applicable.

\section{Competing interests}

The authors declare that they have no competing interests.

\section{References}

1. Al-Achkar W, Wafa A,Liehr T, Klein E and Moassass F: Detailed analysis of an $\operatorname{idic}(\mathrm{Y})(\mathrm{q} 11.21)$ in a mosaic karyotype. Mol Med Rep 6: 293-296, 2012.

2. Li Z, Haines CJ and Han Y: 'Micro-deletions' of the human Y chromosome and their relationship with male infertility. J Genet Genomics 35: 193-199, 2008.

3. Kuroda-Kawaguchi T, Skaletsky H, Brown LG, Minx PJ, Cordum HS, Waterston RH, Wilson RK, Silber S, Oates R, Rozen S and Page DC: The AZFc region of the Y chromosome features massive palindromes and uniform recurrent deletions in infertile men. Nat Genet 29: 279-286, 2001.

4. Repping S, Skaletsky H, Lange J, Silber S, Van Der Veen F, Oates RD, Page DC and Rozen S: Recombination between palindromes $\mathrm{P} 5$ and $\mathrm{P} 1$ on the human $\mathrm{Y}$ chromosome causes massive deletions and spermatogenicfailure. Am J Hum Genet 71: 906-922, 2002.

5. Vogt PH, Edelmann A, Kirsch S, Henegariu O, Hirschmann P, Kiesewetter F, Köhn FM, Schill WB, Farah S, Ramos C, et al: Human Y chromosome azoospermia factors(AZF) mapped to different subregions in Yq11. Hum Mol Genet 5: 933-943, 1996.

6. Hsu LY: Phenotype/karyotype correlations of Y chromosome aneuploidy with emphasis on structural aberrations in postnatally diagnosed cases. Am J Med Genet 53: 108-140, 1994.

7. Jaruzelska J, Korcz A, Wojda A, Jedrzejczak P, Bierla J, Surmacz T, Pawelczyk L, Page DC and Kotecki M: Mosaicism for $45, X$ cell line may accentuate the severity of spermatogenic defects in men with AZFc deletion. J Med Genet 38: 798-802, 2001.

8. Reshmi SC, Miller JL, Deplewski D, Close C, Henderson LJ, Littlejohn E, Schwartz S and Waggoner DJ: Evidence of a mechanism for isodicentric chromosome $Y$ formation in a $45, X / 46, X, \operatorname{idic}(Y)(p 11.31) / 46, X, \operatorname{del}(Y)(p 11.31)$ mosaic karyotype. Eur J Med Genet 54: 161-164, 2011.

9. Hernando C, Carrera M, Ribas I, Parear N, Baraibar R, Egocue J and Fuster C: Prenatal and postnatal characterization of Y chromosome structural anomalies by molecular cytogenetic analysis. Prenat Diagn 22: 802-805, 2002.

10. Jakubowski L, Jeziorowska A, Constantinou $M$ and Kałuzewski B: Molecular analysis of Y chromosome long arm structural instability in patients with gonadal dysfunction. Clin Genet 57: 291-295, 2000.

11. DesGroseilliers M, Beaulieu Bergeron M, Brochu P, Lemyre E and Lemieux N: Phenotypic variability in isodicentric Y patients: Study of nine cases. Clin Genet 70: 145-150, 2006.

12. Lee J, Park JK, Kim DS, Lee HS, Choi SI and Cho YG: Detailed analysis of isodicentric $\mathrm{Y}$ in a case with azoospermia and 45,x/46,x,idic(Y) mosaicism. Ann Clin Lab Sci 45: 206-208, 2015.

13. Becker RE and Akhavan A: Prophylactic bilateral gonadectomy for ovotesticular disorder of sex development in a patient with mosaic 45,X/46,X,idic(Y)q11.222 karyotype. Urol Case Rep 5: 13-16, 2016.

14. Shinawi M, Cain MP, Vanderbrink BA, Grignon DJ, Mensing D, Cooper ML, Bader $P$ and Cheung SW: Mixed gonadal dysgenesis in a child with isodicentric Y chromosome: Does the relative proportion of the 45,X line really matter? Am J Med Genet A 152A: 1832-1837, 2010.

15. Jiang Y, Wang R, Li L, Xue L, Deng S and Liu R: Molecular cytogenetic study of de novo mosaic karyotype 45,X/46,X,i(Yq)/46,X,idic(Yq) in an azoospermic male: Case report and literature review. Mol Med Rep 16: 3433-3438, 2017.

16. Si YM, Dong Y, Wang W, Qi KY and Wang X: Hypospadias in a male infant with an unusual mosaic $45, \mathrm{X} / 46, \mathrm{X}, \mathrm{psu}$ idic(Y) (p11.32)/46,XY and haploinsufficiency of SHOX: A case report. Mol Med Rep 16: 201-207, 2017.

17. Zhang L, Ren M, Song G, Zhang Y, Liu XX, Zhang X and Wang J: Prenatal diagnosis of sex chromosomal inversion, translocation and deletion. Mol Med Rep 17: 2811-2816, 2018.

18. Ghionzoli M, Repele A, Sartiani L, Costanzi G, Parenti A, Spinelli V, David AL, Garriboli M, Totonelli G, Tian J, et al: Human amniotic fluid stem cell differentiation along smooth muscle lineage. FASEB J 27: 4853-4865, 2013. 
19. Fishman MC and Schaffner AE: Carotid body cell culture and selective growth of glomus cells. Am J Physiol 246: C106-C113, 1984.

20. Patsalis PC, Skordis N, Sismani C, Kousoulidou L, Koumbaris G, Eftychi C, Stavrides G, Ioulianos A, Kitsiou-Tzeli S Galla-Voumvouraki A, et al: Identification of high frequency of Y chromosome deletions in patients with sex chromosome mosaicism and correlation with the clinical phenotype and Y-chromosome instability. Am J Med Genet A 135: 145-149, 2005.

21. Kotzot D, Dufke A, Tzschach A, Baeckert-Sifeddine IT, Geppert M, Holland H, Florus JM and Froster UG: Molecular breakpoint analysis and relevance of variable mosaicism in a woman with short stature, primary amenorrhea, unilateral gonadoblastoma, and a 46,X,del(Y) (q11)/45,X karyotype. Am J Med Genet 112: 51-55, 2002.

22. DesGroseilliers M, Fortin F, Lafrenière AM, Brochu P, Lemyre E and Lemieux N: Dynamic increase of a 45,X cell line in a patient with multicentric ring $\mathrm{Y}$ chromosomes. Cytogenet Genome Res 115: 90-93, 2006.

23. Telvi L, Lebbar A, Del Pino O, Barbet JP and Chaussain JL: 45,X/46,XY mosaicism: Report of 27 cases. Pediatrics 104: 304-308, 1999.
24. Lange J, Skaletsky H, van Daalen SK, Embry SL, Korver CM, Brown LG, Oates RD, Silber S, Repping S and Page DC: Isodicentric Y chromosomes and sex disorders as byproducts of homologous recombination that maintains palindromes. Cell 138: 855-869, 2009.

25. Lahn BT and Page DC: Functional coherence of the human Y chromosome. Science 278: 675-680, 1997.

26. Skaletsky H, Kuroda-Kawaguchi T, Minx PJ, Cordum HS, Hillier L, Brown LG, Repping S, Pyntikova T, Ali J, Bieri T, et al: The male-specific region of the human Y chromosome is a mosaic of discrete sequence classes. Nature 423: 825-837, 2003

27. Delbridge ML, Lingenfelter PA, Disteche CM and Graves JA: The candidate spermatogenesis gene RBMY has a homologue on the human X chromosome. Nat Genet 22: 223-224, 1999.

28. Tsui S, Dai T, Roettger S, Schempp W, Salido EC and Yen PH: Identification of two novel proteins that interact with germ-cell-specific RNA-binding proteins DAZ and DAZL1. Genomics 65: 266-273, 2000. 